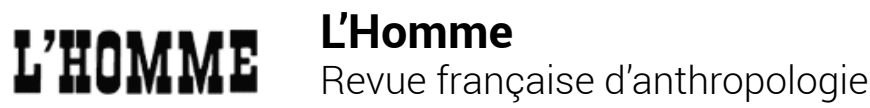

175-176 | juillet-septembre 2005

Vérités de la fiction

Niels Gutschow et al., eds., Sacred Landscape of the Himalaya

Actes du colloque tenu à Heidelberg, 25-27 mai 1998. Wien, Verlag der Österreichischen Akademie der Wissenschaften. 2003, 212 pages

\section{Patrick Kaplanian}

\section{(2) OpenEdition}

1 Journals

\section{Édition électronique}

URL : http://journals.openedition.org//homme/2071

DOI : 10.4000//homme.2071

ISSN : 1953-8103

Éditeur

Éditions de l'EHESS

\section{Édition imprimée}

Date de publication : 15 octobre 2005

Pagination : $542-544$

ISBN : 2-7132-2035-1

ISSN : 0439-4216

Référence électronique

Patrick Kaplanian, «Niels Gutschow et al., eds., Sacred Landscape of the Himalaya », L'Homme [En ligne], 175-176 | juillet-septembre 2005, mis en ligne le 30 novembre 2006, consulté le 24 septembre 2020. URL : http://journals.openedition.org/lhomme/2071; DOI : https://doi.org/10.4000/lhomme.2071

Ce document a été généré automatiquement le 24 septembre 2020.

(c) École des hautes études en sciences sociales 


\section{Niels Gutschow et al., eds., Sacred Landscape of the Himalaya}

Actes du colloque tenu à Heidelberg, 25-27 mai 1998. Wien, Verlag der Österreichischen Akademie der Wissenschaften. 2003, 212 pages

\section{Patrick Kaplanian}

\section{RÉFÉRENCE}

Niels Gutschow et al., eds., Sacred Landscape of the Himalaya. Actes du colloque tenu à Heidelberg, 25-27 mai 1998. Wien, Verlag der Österreichischen Akademie der Wissenschaften. 2003, 212 p., bibl., index, ill., cartes.

1 LE THÈME de ce colloque, qui s'est tenu à Heidelberg en mai 1998 sous la direction de quatre grands spécialistes de l'Himalaya (Niels Gutschow, Axel Michaels, Charles Ramble et Ernst Steinkellner), est l'espace. Il s'agit plus particulièrement de l'espace sacré, quoique - cela ressort de la lecture du livre - il n'y ait pas d'opposition ou de distinction entre espace sacré et espace profane. Le ton est d'ailleurs donné dès l'introduction.

2 Axel Michaels montre en effet comment l'espace « indigène » est différent de celui de notre géométrie. Ainsi le haut et le bas s'opposent avec d'autres paires d'oppositions qui ne sont pas sans rappeler les homologies de Claude Lévi-Strauss: ainsi le haut, domaine des lha (divinités) vs le bas, domaine des hommes, mais aussi le haut domaine sauvage de rochers et de glaciers vs le bas, domaine des champs cultivés. Mais si "haut» peut désigner le domaine des dieux, tout comme un monde sauvage dans lequel il est impossible de vivre, les paires dieux (lha)/hommes et monde sauvage/ champs cultivés impliquent donc quelque chose de contradictoire. C'est qu'il y a plusieurs espaces: sacré, vécu, etc. et non pas un seul et unique espace, continu, homogène, isotrope comme dans notre géométrie.

3 Marc Dujardin présente deux cas de démolition et de reconstruction à Rukubji un village du Bhoutan central. Le village est très minutieusement décrit: le répertoire 
architectural religieux y est relativement limité : un lhakhang («chapelle»), quatre chorten (qui sont à la fois des reliquaires, des constructions votives, des représentations du corps de Bouddha et de la religion bouddhique), un tsenkhang (orth. btsan-khang, autel extérieur de la divinité tutélaire du village) et quinze lhubang (orth. klu-khang ou klu-bang, autel extérieur des divinités du sous-sol et de la fertilité). Les événements qu'il décrit amenèrent les villageois à reconstruire le lhakhang et à déplacer le tsenkhang. Mais «transfert» n'est pas «restauration » et il ne s'agit pas de reconstruire à l'identique par dévotion pour les monuments anciens. L'auteur montre que religion et architecture ne sont pas figées mais que, à partir de principes de base, le système s'adapte sans arrêt.

4 Ada Gansach examine l'aspect spatial de trois rituels dans trois villages du nord-ouest du Népal: le losar (nouvel an) à Nyimatang (vallée de Humla), un rituel contre la sécheresse à Tugkhyu (région du Dolpo) et targya (mtha'-rgyas), un rituel après la récolte en automne à Braga (district de Manang). Tous les trois ont en commun d'inclure une procession, mais les parcours à travers champs et maisons sont très différents. L'auteur rattache ces différences à des évolutions historiques divergentes.

5 John Harrison reconstitue le premier plan de Lo Manthang, la capitale du Mustang, une principauté adossée à la frontière chinoise du Népal auquel elle appartient maintenant et qui fut longtemps indépendante. Il montre que l'agglomération n'avait pas été conçue pour être une place séculière, une simple ville, mais un centre cérémoniel et un symbole du pouvoir royal.

6 Franz-Karl Ehrhard choisit un angle original en prenant pour sujet les itinéraires de pèlerinage de deux lamas (moines de très haut rang) au Mustang, Orgyen Chöpel (né en 1755) et Sangye Sangpol (né en 1891). Il conclut que leurs pérégrinations avaient moins pour objectif de visiter des lieux saints, but avoué, que d'en créer.

7 Si F.-K. Ehrard étudie les pèlerinages des lamas, William Sax analyse, quant à lui, ceux des divinités elles-mêmes, et ce, au Garhwal, entre Népal et Ladakh, où il arrive que l'on promène les statues de divinités hindoues sur des palanquins. Il montre que le pèlerinage de certaines divinités aux sources du Gange est récent et coïncide avec l'arrêt des sacrifices sanglants. Il s'agit pour les Gahrwali de s'intégrer à la société indienne et hindoue des plaines et de ne plus se sentir méprisés par elle. Notons que c'est la seule contribution du livre où il est question de l'hindouisme et non du bouddhisme.

8 Niels Gutschow et Charles Ramble s'intéressent à quatre villages de Baragaon (Bas-Lo, district du Mustang, au nord-ouest du Népal) qui sont décrits, cartographiés et photographiés avec beaucoup d'acuité. Ils montrent que la démarcation que fait un village entre l'interne et l'externe n'est jamais absolue et que tel objet « externe » peut être reclassé « interne " pour le besoin des circonstances. Ils donnent des exemples de déplacement des limites et décrivent aussi l'axe vertical par lequel transitent les bienfaits divins.

9 Penchons-nous pour finir sur les trois contributions consacrées au Ladakh, tout à l'ouest de l'Himalaya indien. Ceux qui ont lu l'excellent travail de Reinhard Herdick sur le village de Yantang dans les actes du VIII ${ }^{e}$ colloque sur le Ladakh ${ }^{1}$ ne sont pas déçus par la suite. L'auteur décrit, cette fois-ci, avec une précision de géomètre, la localisation des nyitho (qui indiquent le lever et le coucher du soleil aux solstices), la répartition spatiale des thatho (autels extérieurs des divinités tutélaires de village qui, au Bhoutan, s'appellent tsendos), des lhubang et des tsandos (btsan-mdos, qui au Ladakh désignent des 
repoussoirs de couleur rouge pour éloigner des démons appelés tsan), aussi bien dans les champs que sur les maisons, la répartition des tshatshakhang (où l'on place des médaillons faits d'argile et de cendres des défunts). Et si tous ces éléments sont connus en tant que tels, il fallait un architecte pour en étudier aussi précisément la distribution spatiale. L'auteur étend ensuite son étude au monastère tout proche de Rizong et à la nonnerie de Chulican. Il montre comment tout l'ensemble est ordonné en cercles concentriques, avec entre eux des seuils, et aussi du haut vers le bas, selon les points cardinaux, etc., le tout formant un ensemble très complexe mais cohérent. Un bel exemple d'ethno-urbanisme.

Après le travail très riche du même Reinhard Herdick dans les actes du VII colloque sur le Ladakh ${ }^{2}$ et sur le village de Lamayuru, Amandus Vanquaille et Hilde Vets rouvrent le dossier. Après un tableau assez exhaustif du village et de la gonpa (le monastère), ils se tournent vers un phénomène curieux qui, à ma connaissance, n'a jamais été étudié : celui des ombres et des lumières. Ainsi, dans la période autour du solstice d'été, le lhatho est le premier illuminé par le soleil, puis vient la gonpa, puis le village. Et il n'y a pas de maisons dans la zone qui reste toute la journée dans l'ombre. Un phénomène analogue se reproduit le soir. Les observations sont intéressantes, mais la conclusion n'est pas à la hauteur. Il y avait, écrivent les auteurs, au vII siècle, un important culte du soleil au Cachemire. Et ce qu'ils ont découvert à Lamayuru n'en serait que les restes. Nous voilà ramenés à un diffusionnisme un peu dépassé ! C'est une chose de savoir qu'il y avait un culte solaire au Cachemire (à deux semaines de marche de là) à une certaine époque, et de constater le phénomène décrit à Lamayuru ensuite. C'en est une autre d'établir un rapport de cause à effet. Il faut dans ce cas retracer l'itinéraire.

Kim et Niels Gutschow nous proposent un travail très complet sur le hameau de Rinam (5 khangpa ou maisonnées) au Zanskar, (une sous-région du Ladakh, sorte de cirque encaissé). C'est un chef-d'œuvre d'ethnographie. Les cinq familles sont décrites en détail (il faut avoir un crayon et un papier à portée de main), puis les canaux, les champs irrigués canal par canal, les rotations de l'eau et enfin la distribution des champs entre les khangchen (maisons principales) et les khangchung (maisons secondaires ou habitent les vieux parents ou un frère cadet, et qui ne sont pas indépendantes). La dernière partie élargit l'enquête à l'espace autour. On y apprend que le corps d'une démone, «cloué » par des monastères, couvre le Zanskar central. Comme tout travail intéressant, celui des Gutschow suscite de nouvelles questions. On constate ainsi que pour la maison Yogma (p. 121) tous les champs de la khangchen sont regroupés au nord et ceux de la khangchung au sud. Mais tel n'est pas le cas pour les maisons Hilma et Gongma où la distribution semble aléatoire. Comment se fait une répartition des champs entre khangchen et khanchung lorsqu'une khangchung est créée ? Cela a-t-il été étudié? Autre question: pourquoi retrouve-t-on l'histoire, en fait tibétaine, de la démone, « clouée » au Zanskar, alors qu'elle est, semble-t-il, absente de la vallée de l'Indus entre le Zanskar et le Tibet?

Dans l'ensemble, il s'agit d'analyses très fouillées et très denses sur un sujet extrêmement vaste et complexe.

13 Un dernier mot: c'est un livre absolument superbe, abondamment illustré de magnifiques photos en couleurs, fourni de dessins, schémas, cartes et plans. L'analyse de la cartographie, très riche, permet au lecteur de suivre de très près les textes et de se faire ses propres opinions. 


\section{NOTES}

1. . Sous la direction de M. Van Beek, K. Brix Bertelsen \& P. Pedersen, Ladakh, Culture, History and Development between Himalaya and Karakoram. Proceedings of the Eight Colloquium of the International Association for Ladakh Studies held at Moesgaard, Aarhus University, 5-8 June 1997, Aarhus, Aarhus University Press, 1999 (« Recent Research on Ladakh 8 »).

2. . T. Dodin \& H. Räther, eds, Proceedings of the Seventh Colloquium of the International Association of Ladakh Studies, Bonn/Sankt Augustin, 12-15 juin 1995, Ulm, Ulmer Kulturanthropologie Schriften, Band 9, 1997 (« Recent Research on Ladakh $7 »)$. Voir mon compte rendu dans L'Homme, 2002, $164: 173$ sq. 